\title{
Identification and characterization of bioactive compound berberine in the Berberis vulgaris root extract using HR-LC-MS analysis
}

\begin{abstract}
Berberis vulgaris $L$. is a shrub of family Berberidaceae produces in Asia and Europe. A broad range of medicinally and nutritionally important phytochemical components have been isolated from $B$. vulgaris, which are of medicinal value. Berberine is the main alkaloid isolated from $B$. vulgaris. Chromatography was performed on an Acquity UPLC system (Waters) equipped with a HSS T3 column18 using mixture of water (containing $0.1 \%$ formic acid) and acetonitrile $(30: 70 \mathrm{v} / \mathrm{v})$ as mobile phase. The mass spectrometric detection was performed by selected ion monitoring mode via electrospray ionization in the positive ionisation mode. The results indicate that the approximate berberine content in the $B$. vulgaris root extract, as determined by the proposed method was $0.7266 \mathrm{mg} \mathrm{ml}-1$. The fully validated HR-LC/MS method can be successfully applied for the determination of berberine in $B$. vulgaris, which can be used as a potent therapeutic agent for the treat of hypopigmentary disorders
\end{abstract}

Volume 7 Issue 4 - 2018

\author{
Ishrat Naaz,' Sharique A Ali² \\ 'Research Scholar, Saifia College of Science, India \\ ${ }^{2}$ Professor \& Head Department of Zoology and Biotechnology, \\ Saifia College of Science, India
}

Correspondence: Ishrat Naaz, Research Scholar, Saifia College
of Science, Bhopal MP, 46200I, India Email ishrat.naaz03@gmail.com

Received: June 23, 2018| Published: August 27, 2018

Keywords: berberis vulgaris, alkaloid, berberine, hypopigmentation

\section{Introduction}

Ayurvedic medicines and formulations acquired from ancient Indian herbal systems are well-known for their various important applications. Recently, the interest and investigation in medicinal plants have augmented immensely. Berberis vulgaris L. is a shrub of family Berberidaceae produces in Asia and Europe; it is named in English as barberry. ${ }^{1}$ It is a deciduous shrub growing up to $4 \mathrm{~m}$ high. The leaves are small oval, $2-5 \mathrm{~cm}$ long and $1-2 \mathrm{~cm}$ broad, with a serrated margin; they are borne in clusters of 2-5 together, subtended by a three-branched spine $3-8 \mathrm{~mm}$ long. The flowers are yellow, 4-6mm across, produced on $3-6 \mathrm{~cm}$ long panicles in late spring. The fruit is an oblong red berry $7-10 \mathrm{~mm}$ long and $3-5 \mathrm{~mm}$ broad, ripening in late summer or autumn; they are edible but very sour, and rich in Vitamin C. ${ }^{2}$ The plant has been used widely for centuries for the treatment of various ailment. A broad range of medicinally and nutritionally important phytochemical components have been isolated from various parts of the plants such as alkaloids, saponins, cardioactive glycosides, anthocyanins, tanins, carbohydrates, protein, lipid, vitamins, fiber contents, $\beta$ carotein. Phytic acid and phytate phosphorus etc.

Plant is reported to possess twenty two alkaloid from roots, stems, leaves and fruits, which are of medicinal value. ${ }^{4}$ The alkaloid content differs from the different areas, different species and different organs. ${ }^{5}$ Bereberine is the key alkaloid components with an isoquinolonic nucleus isolated from the roots and bark of B. vulgaris. ${ }^{6}$ It is an isoquinoline plant alkaloid belongs to the structural class protoberberines. Berberine is the one of the most studied alkaloid among the various naturally occurring protoberberine. It is reported to possess various pharmacological actions including antibacterial, anti-pyretic, anti-hepatotoxic, anti-cancer, anti-lipidemic, antihyperglycemic, anti-oxidant agent. ${ }^{6-8}$ It has been used in some cases like diarrhoea, haemorrhoids, osteoporosis, leishmaniasis, eye and ear infections, jaundice, kidney and gall bladder stones, wound healing, skin diseases and malaria fever. ${ }^{2}$

Mukherjee et al. ${ }^{9}$ have reported that decoction of roots of $B$. vulgaris can be used for skin troubles. Recently we have investigated that the berberine as its helps in pigment dispersal in the skin melanophores of adult Bufo melanostinctus via $\beta$-2-adrenergic receptor. ${ }^{10,11}$ Skin darkening effect of berberine was further supported by ultrastructural analysis of skin melanophores of $B$. melanostinctus. ${ }^{12}$ These studies suggest that the berberine could have been used as potential therapeutic agent for the treatment of various hypopigmentary disorders like vitiligo. ${ }^{11,13}$

Considering the great therapeutic potential of this plant, it was of great importance to qualitatively and quantitatively evaluate the percentage of berberine content in the root extract of B. vulgaris. A number of analytical methods have recently been reported for the determination of berberine content in the root extract of $B$. vulgaris. The chromatographic technique provides an excellent precision for the routine determination of the alkaloid content of $B$. vurgaris roots extract. The separation and purification of berberine from $B$. vulgaris by conventional methods such as column chromatography and high-performance liquid chromatography (HPLC) is tedious, time consuming and usually requires multiple chromatography steps. ${ }^{14}$ LCMS detection is one of the most powerful analytical tools for organic compound analysis as quantitative and qualitative data can be obtained easily with limited instrument optimisation. In present study aims to isolate berberine from $B$. vulgaris and an attempt has been made to develop and validate HPTLC and LC/MS method for the analysis of berberine content in the root extract of $B$. vulgaris, which would be highly sensitive, having high resolution, shorter retention time and reproducibility.

\section{Materials and method}

Analytical grade ethanol and methanol were purchased from Qualigens Fine Chemicls, Mumbai, India. Formic acid (HPLC grade) and Acetonitril (HPLC grade) was procured from Sigma Aldrich (USA). Berberine Chloride Dihydrate was obtained from Alfa Aesar (USA). Daruhaldi or the root of Berberis vulgaris was purchased from local market of Bhopal. The plant sample was identified and authenticated by Dr. Zia ul Hasan, Botanist Department of Botany, Saifia Science College, Bhopal. The voucher specimen (No.:452/Bot/ Saifia/14) was deposited at the botanical herbarium at Saifia Science 
College, Bhopal (M.P.), India. Preservation of plants material was done according to standard protocol following the literature: technical reports and manuals.

\section{Plant material and preparation of extract}

Soxhlet extraction method was performed for the preparation of crude extracts containing high content of berberine from the root of $B$. vulgaris. For preparation of the alcoholic extracts of $B$. vulgaris, $100 \mathrm{~g}$ of roots were dried at room temperature in dark and then crushed into coarse powder. The powdered material was then soaked in $100 \mathrm{ml}$ of $80 \%$ ethanol overnight and then was exhaustively extracted with $80 \%$ ethanol $(100 \mathrm{ml} \mathrm{X} 2)$ in a Soxhlet apparatus at $80^{\circ} \mathrm{C}$ for $72 \mathrm{~h}$. The crude extract was filtered and evaporated to dryness on a water bath set at $100^{\circ} \mathrm{C}$. The dried residue of crude extract was cooled in desiccators for $30 \mathrm{~min}$, then filtered via disk of filter paper and accurately weighed for analysis. ${ }^{15}$

\section{Preparation of standard and sample solutions}

A stock solution of berberine was prepared by dissolving $4.8 \mathrm{mg}$ of standard berberine chloride dihydrate (equivalent to $3.96 \mathrm{mg}$ of berberine) in $10 \mathrm{ml}$ methanol. The standard solution of berberine was prepared by diluting the stock solution to obtain the concentration of $99 \mu \mathrm{g} / \mathrm{ml}$. The sample solution was prepared by weighing dried extracts $(10 \mathrm{mg})$, dissolving in each extracting solvent and adjusting to $10 \mathrm{ml}$.

\section{High resolution liquid chromatography mass spectrometry (HR-LC/MS) analysis}

The total berberine content in the root extract of $B$. vulgaris was quantitatively determined by HR-LC/MS analysis. Chromatographic separations were performed on an Acquity UPLC system (Waters) equipped with a HSS T3 column $18(100 \times 1.0 \mathrm{~mm}$, particle size $1.8 \mu \mathrm{m}$; Waters); mobile phase consist of water (containing $0.1 \%$ formic acid) and acetonitrile $(30: 70 \mathrm{v} / \mathrm{v})$; applying the flow rate of $0.45 \mathrm{~mL} \mathrm{~min}^{-1}$; $0-0.5 \mathrm{~min}$. The injection volume was $3.1 \mu \mathrm{L}$ (full loop injection). The thermo stated auto sampler was kept at $4^{\circ} \mathrm{C}$.

The mass spectrometer operated using an electrospray ionisation (ESI) source in positive mode and was set for isolation and fragmentation of the berberine molecular ion. Eluted compound was detected from m/z 50 to 3000 using a Micro-TOF-Q hybrid quadrupole time-of-flight mass spectrometer (Bruker micrOTOF-Q-II Daltonics) equipped with an Apollo II electrospray ion source in positive ion modes. MS operating condition were optimized as follows: nebulizer gas: nitrogen; drying gas flow: $7.0 \mathrm{~L} / \mathrm{min}$; nebulizer pressure: 1.2 bar; capillary voltage: $4500 \mathrm{~V}$; end plate offset: $-500 \mathrm{~V}$; collision cell RF:130Vpp. drying gas temperature: $200^{\circ} \mathrm{C}$. System control and data acquisition were controlled by Bruker Compass Data Analysis 4.0.

\section{Results and discussion}

B. vulgaris is reported to have wide range of pharmacological activities. It is used medicinally in all the traditional medical systems and has a history of usage in Ayurvedic, Iranian as well as Chinese medicines dating back at least 3000 years. ${ }^{16}$ Though numbers of RP-HPLC, LC-MS-MS, UPLC-MS/MS, LC MS/MS Q-tof, etc. techniques have been developed for the qualitative and quantitative estimation of berberine in Tinospora cordifolia, various formulation, rat and rabbit plasma. ${ }^{17-21}$ But there is no report on the quantitative screening of berberine content in the root extract of B. vulgaris using HR-LC/MS analysis. It has been observed that the vacuum evaporated extract of $B$. vulgaris gave yellowish dark brown extract which was subjected to HR-LC/MS analysis for proper qualitative as well as quantitative identification of berberine. In order to identify and quantify the main alkaloid from the root extract of $B$. vulgaris by HR-LC/MS analysis standard berberine chloride dihydrate was used.

The UPLC chromatogram of detected compound i.e. berberine in the root extract of $B$. vulgaris and standard berberine is shown in Figure 1. It is obvious that the composition of mobile phase also affects the separation and ionization of phytocompounds. Therefore, in order to boost the reproducibility, formation and sensitivity of the analysis, several additives in different concentration were incorporated to the mobile phase like formic acid, acetic acid and ammonium acetate. It was observed that the addition of $0.1 \%$ formic acid enhance the reproducibility and sensibility of the analysis as single, sharp and symmetrical peaks were obtained The average recovery of berberine at different level was noticed to be $98.75 \%$. The obtained LC-MS total ion chromatograms, UV characteristics and extracted ion chromatogram of $B$. vulgaris root extract along as well as standard berberine are depicted in Figure 1. Many compounds were observed in the UPLC chromatogram of B. vulgaris, but the purity of berberine was reported as $45 \%$ based on UPLC peak area.

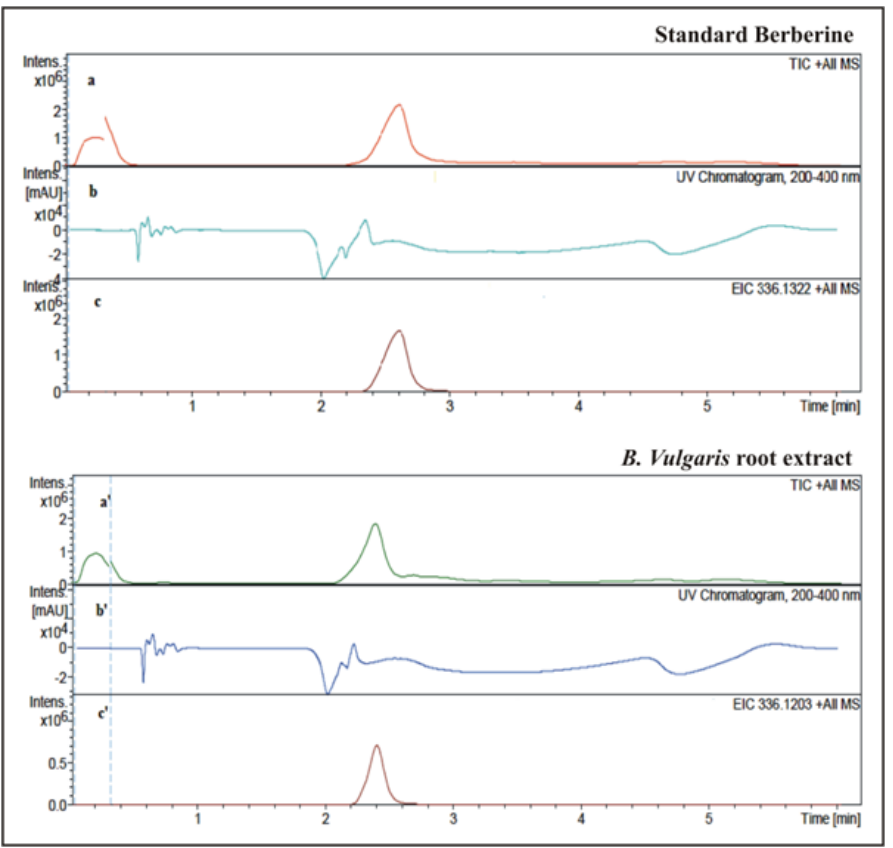

Figure I Comparison of standard berberine and root extract of $B$. vulgaris via UPLC-HRLC-MS analysis (a-a') Total ion counts vs. acquisition time (min); (bb') UV chromatogram; (c-c') Extracted ion counts vs. Acquisition time (min).

The identities, retention time and observed molecular and protonated ions for individual components are depicted in Figure 2. It was observed that the highest peak was obtained at retention time 2.3$2.5 \mathrm{~min}$ belonging to the phytocompound berberine in $B$. vulgaris. With the standard reference graph, the berberine compound is elucidated using the molecular weight. Quantification of berberine was based on the sum of ions with $\mathrm{m} / \mathrm{z}=36.1226$ and 453.3392 from the MS spectrum of the parent ion Figure 3. MS/MS chromatogram showed similar fragmentation patter for both the standard berberine and compound from the root extract of $B$. vulgaris. Berberine concentration in the $B$. vulgaris root extract determined by HR-LC/MS was $0.7266 \mathrm{mgml}^{-1}$ of extract. The most important phytocompound identified in the root 
extract of $B$. vulgaris is berberine, which is known to exhibit various outstanding pharmacological activity. ${ }^{1,22}$

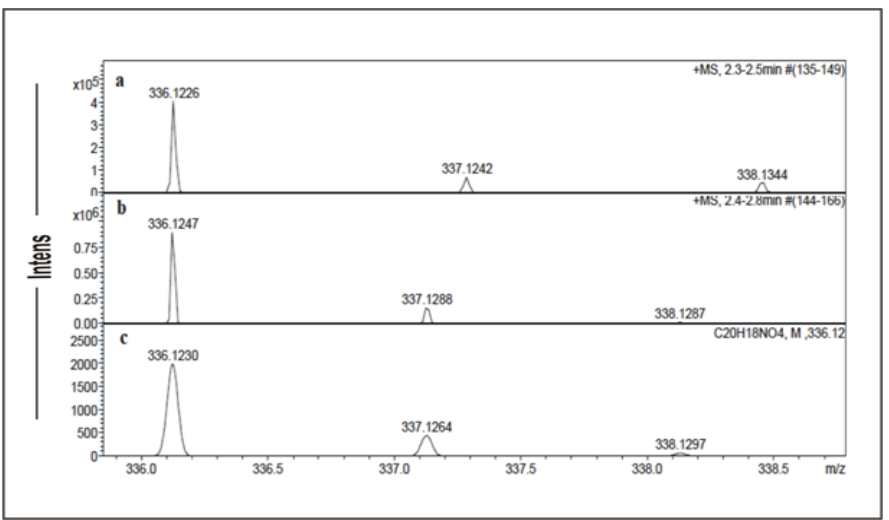

Figure 2 Comparative analysis of LC-MS base peak intensity chromatograms derived from positive ionization mode $(\mathrm{m} / \mathrm{z}=50-3000)$. Count vs. Mass-tocharge (m/z): (a) B. vulgaris root extract (b) standard Berberine (c) theoretical composition.

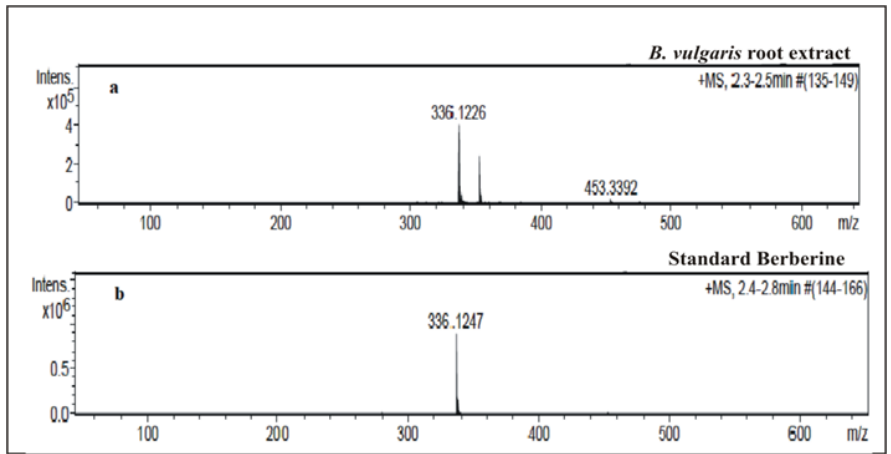

Figure 3 Full scan MS of (a) B. vulgaris root extract (b) standard Berberine in mobile phase.

The result showed that the exact mass of berberine content of B. vulgaris $\left([\mathrm{M}-\mathrm{H}]^{+} \mathrm{m} / \mathrm{z} 336.1226\right)$ was identical to that of the standard berberine $\left([\mathrm{M}-\mathrm{H}]^{+} \mathrm{m} / \mathrm{z} 336.1247\right)$, matching the theoretical composition of $\mathrm{C}_{20} \mathrm{H}_{18} \mathrm{NO}_{4}\left([\mathrm{M}-\mathrm{H}]^{+} \mathrm{m} / \mathrm{z}\right.$ 336.1230) (Figure 2). The UV absorbance spectra of sample (root extract of B. vulgaris) and berberine during LC-UV-MS were recorded and absorbance maxima were noticed at $267 \mathrm{~nm}$ (Figure 1). The inclusive results of present investigation has proved that HR-LC/MS is the highly precise analytical technique which can be extensively used for isolation and quantification of berberine content in the root extract of $B$. vulgaris.

\section{Conclusion}

The present investigation described and justified a simple, sensitive HR-LC/MS technique for the characterization and quantitative estimation of the main alkaloid i.e. berberine content in the root extract of $B$. vulgaris. The HR-LC/MS analysis was implicated for the detailed studies of berberine in the extract. After incorporating required modification, the present analytical method is proposed to determine the alkaloid content in the various samples.

\section{Acknowledgements}

The authors are thankful to the Secretary and Principal of Saifia Science College, Bhopal, India for providing the necessary facilities.

\section{Conflicts of interest}

The authors have no conflict of interest to declare.

\section{References}

1. Tamilselvi S, Balasubramani SP, Venkatasubramanian P, et al. A review on the pharmacognosy and pharmacology of the herbals traded as 'daruharidra'. Int J Pharm Bio Sci. 2014;5(1):556-570.

2. Abd El-Wahab AE, Ghareeb DA, Sarhan EE, et al. In vitro biological assessment of Berberis vulgaris and its active constituent, berberine: antioxidants, anti-acetylcholinesterase, anti-diabetic and anticancer effects. BMC Complement Altern Med. 2013;13:218

3. Akbulut M, Çalişir S, Marakoğlu T, et al. Some physicomechanical and nutritional properties of barberry (Berberis vulgaris L.) Fruits. Journal of Food Process \& Engineering. 2009;32(4):497-511.

4. Arayne, MS, Sultana N, Bahadur SS. The berberis story: Berberis vulgaris in therapeutics. Pak J Pharm Sci. 2007;20(1):83-92.

5. Di DL, Liu YW, Ma ZG, et al. Determination of four alkaloids in Berberis plants by HPLC. Zhongguo Zhong Yao Za Zhi. 2003;28(12):1132-1134.

6. El-Salama MA, Mekky H, El-Naggar EMB, et al. Hepatoprotective properties and biotransformation of berberine and berberrubine by cell suspension cultures of Dodonaea viscosa and Ocimum basilicum. South African Journal of Botany. 2015;97:191-195.

7. Maithani A, Parcha V, Kumar D. Quantitative estimation of berberine content of Berberis asiatica from different altitude of Garhwal Himalaya. Asian J Pharm Clin Res. 2004;7( Suppl 1):165-167.

8. Pashaee M, Shiravi A, Hojati V. The effect of hydroalcoholic extract of Berberis vulgaris on wound healing of diabetic wistar rats. Journal of Chemical Health Risks. 2016;6(4):319-325.

9. Mukherjee PK, Maitya N, Nemaa NK, et al. Bioactive compounds from natural resources against skin aging. Phytomedicine. 2011;19(1):64-73.

10. Ali SA, Naaz I, Choudhary RK. Berberine induced pigment dispersion in Bufo melanostictus melanophores by stimulation of beta-2 adrenergic receptors. J Recept Signal Transduct Res. 2014;34(1):5-20.

11. Ali SA, Naaz I, Zaidi KU, et al. Recent updates on melanocyte biology and the use of promising bioactive compounds for the treatment of hypopigmentary disorders: A review. Mini Rev Med Chem. 2017;17(9):785-798.

12. Ali SA, Naaz I. Understanding the ultrastructural aspects of berberine induced skin darkening activity in the toad, Bufo melanostictus melanophores. Journal of Microscopy and Ultrastructure. 2015a;3(4):210-219.

13. Ali SA, Naaz I. Current challenges in understanding the story of skin pigmentation: Bridging the morpho-anatomical and functional aspects of mammalian melanocytes. In: Kunihiro Sakuma, editor. Muscle Cell and Tissue. Europe: InTech Open House; 2015b:262-285.

14. Nargis Khan, Sharique A Ali. RLC-MS analysis of isoliquiritigenin from the root extract of Glycyrrhiza glabra for developing a novel depigmenting agent. Biosci Biotech Res Comm. 2014;7(1):89-93.

15. Rojsanga P, Gritsanapan W, Suntornsuk L. Determination of berberine content in the stem extracts of Coscinium fenestratum by TLC densitometry. Med Princ Pract. 2006;15(5):373-378.

16. Timothy CBN, Gregory S, Kelly ND. Berberine: therapeutic potential of an alkaloid found in several medicinal plants. Altern Med Rev. 1997;2(2):94-103

17. Pradhan D, Biswasroy P, Suri KA. Isolation of Berberine from Berberis vulgaris Linn and standardization of aqueous extract by RP-HPLC. International Journal of Herbal Medicine. 2013;1(2):106-111. 
18. Yan R, Wang Y, Liu Y, et al. Comparative pharmacokinetics of berberine, palmatine and jatrorrhizine in rat plasma after oral administration of rhizoma coptidis and Zuojinwan using liquid chromatography-tandem mass spectrometry. Iran J Pharm Res. 2012;11(3):949-957.

19. Li G, Yang F, Liu M, et al. Development and application of a UPLCMS/MS method for simultaneous determination of fenofibric acid and berberine in rat plasma: application to the drug-drug pharmacokinetic interaction study of fenofibrate combined with berberine after oral administration in rats. Biomed Chromatogr. 2016;30(7):1075-1082.

20. Li X, Liu H, Li J, et al. Simultaneous determination of berberine and palmatine in rabbit plasma by LC-MS-MS and its application in pharmacokinetic study after oral administration of coptidis and coptidisgardeniae couple extract. Chromatographia. 2009;70(7/8):1113-1119.
21. Mohan MC, Abhimannue AP, Kumar P. Identification and characterization of berberine in Tinospora cordifolia by liquid chromatography quadrupole time of flight mass spectrometry (LC MS/MS Q-tof) and evaluation of its anti-inflammatory potential. Pharmacogn J. 2017;9(3):350-355.

22. Parvu M, Parvu AE, Craciun $\mathrm{C}$, et al. Changes in botrytis cinerea conidia caused by Berberis vulgaris Extract. Not Bot Hort Agrobot Cluj. 2010;38(3):15-20. 